\title{
STAT3 is a key molecule in the oncogenic behavior of diffuse intrinsic pontine glioma
}

\author{
JINJU PARK ${ }^{1}$, WOOCHAN LEE ${ }^{2}$, SANGIL YUN ${ }^{1}$, SAET PYOUL KIM ${ }^{1}$, KYUNG HYUN KIM $^{1,3}$, \\ JONG-IL KIM ${ }^{2}$, SEUNG-KI KIM ${ }^{3}$, KYU-CHANG WANG ${ }^{1,3}$ and JI YEOUN LEE ${ }^{1,3}$ \\ ${ }^{1}$ Neural Development and Anomaly Laboratory, Department of Anatomy and Cell Biology; \\ ${ }^{2}$ Department of Biochemistry and Molecular Biology, Seoul National University College of Medicine; \\ ${ }^{3}$ Division of Pediatric Neurosurgery, Seoul National University Children's Hospital, Seoul 03080, Republic of Korea
}

Received September 27, 2019; Accepted April 17, 2020

DOI: 10.3892/ol.2020.11699

\begin{abstract}
Diffuse intrinsic pontine glioma (DIPG) is one of the most lethal childhood brain tumors. This tumor is unique because it is detected exclusively in the ventral pons of patients aged between 6 and 7 years, which suggests a developmental nature of its formation. Signal transducer and activator of transcription 3 (STAT3) is a critical molecule for the differentiation of neural stem cells into astrocytes during neurodevelopment. Additionally, STAT3 is associated with oncogenesis and the epithelial-mesenchymal transition (EMT) in various types of tumor. In recent years, several studies have demonstrated the oncogenic role of STAT3 in high-grade gliomas. However, the role of STAT3 in DIPG at the cellular level remains unknown. To assess the possible association between gliogenesis and DIPG, the expression levels of various molecules participating in the differentiation of neural stem cells were compared between normal brain control tissues and DIPG tissues using open public data. All of the screened genes exhibited significantly increased expression in DIPG tissues compared with normal tissues. As STAT3 expression was the most increased, the effect of STAT3 inhibition in a DIPG cell line was assessed via STAT3 short hairpin (sh)RNA transfection and treatment with AG490, a STAT3 inhibitor. Changes in viability, apoptosis, EMT and radiation therapy efficiency were also evaluated. Downregulation of STAT3 resulted in decreased cyclin D1 expression and cell viability, migration and invasion. Additionally, treatment with STAT3 shRNA or AG490 suppressed the EMT phenotype. Finally, when radiation was administered in combination with STAT3 inhibition,
\end{abstract}

Correspondence to: Professor Ji Yeoun Lee, Neural Development and Anomaly Laboratory, Department of Anatomy and Cell Biology, Seoul National University College of Medicine, 103 Daehakro, Jongno-gu, Seoul 03080, Republic of Korea

E-mail: femiddang@gmail.com

Key words: diffuse intrinsic pontine glioma, signal transducer and activator of transcription 3, epithelial-mesenchymal transition, astrogliogenesis the therapeutic efficiency, assessed by cell viability and DNA damage repair, was increased. The present results suggest that STAT3 is a potential therapeutic target in DIPG, especially when combined with radiation therapy.

\section{Introduction}

Diffuse intrinsic pontine glioma (DIPG) is one of the most fatal malignant pediatric brain tumors (1) and is a major cause of cancer-associated mortality in children (2). The mean overall survival of patients with DIPG is 8-14 months, and the only effective treatment modality is radiation (3). However, clinical outcomes are disappointing, and a combination of various adjuvant chemotherapies has failed to prolong overall survival compared with radiation alone (4). In general, routine tumor biopsy is avoided due to the location of the tumor, and the paucity of tumor tissues has limited active research on the biology and pathogenesis of DIPG. Although several clinical trials for molecular targeted agents are underway (4-6), the focus is on canonical oncogenic signaling pathways, such as the epidermal growth factor receptor and platelet-derived growth factor receptor (PDGFR) signaling pathways. Furthermore, these therapeutic trials are problematic since they are not based on the unique molecular genomic features of DIPG (4).

A number of genetic and epigenetic alterations in DIPG have recently been discovered, revealing a mutation signature that is completely different from that of high-grade gliomas of other regions of the brain. Specifically, the lysine-to-methionine mutation at position 27 of the $\mathrm{H} 3$ histone variant (H3K27M), $\mathrm{H} 3.1 \mathrm{~K} 27 \mathrm{M}$ or $\mathrm{H} 3.3 \mathrm{~K} 27 \mathrm{M}$, was identified in $78 \%$ of DIPGs and was accompanied by activating mutations in PDGFRA or activin A receptor type I (ACVR1) (7-9). The fact that DIPG is detected exclusively in the ventral pons of children aged between 6 and 7 years $(10,11)$ may explain the very different genomic and molecular features compared with other gliomas. Furthermore, the specific peak age and anatomical location suggest abnormalities in postnatal neurodevelopmental mechanisms. Indeed, aberrancy in one of the signaling pathways associated with the differentiation of neural stem cells (NSCs) may cause an imbalance in the number and viability of local cells, and may contribute to growth and neoplastic behavior (12). 
Signal transducer and activator of transcription 3 (STAT3) is a member of the STAT family and has extensive functions, including neural differentiation, in a number of organisms $(13,14)$. STAT3 was originally identified as a mediator of interleukin-6 (IL-6) receptor signaling (15). The receptors for IL-6 utilize gp130, which activates STAT3 via phosphorylation; phosphorylated STAT3 dimerizes and translocates to the nucleus, where it binds to a specific DNA sequence to activate the transcription of genes involved in glial differentiation (16). Furthermore, a previous study demonstrated that STAT3 is essential for astrocyte differentiation (17).

Additionally, STAT3 acts as an oncogene in numerous types of cancer, including breast (18), lung (19) and pancreatic cancer (20). Constitutive activation of STAT3 was first observed to be associated with oncogenic transformation by the viral Src oncoprotein (21). Similarly, other oncogenic tyrosine kinases activate STAT3, resulting in oncogenic transformation (22-24). The contribution of STAT3 to a given tumor phenotype depends on the tissue context. Overall, the role of STAT3 has been studied more in glioma compared with other types of cancer (25-27). In adult glioma, a gain-of-function mutation in STAT3 induces angiogenesis, immunosuppression, invasion and temozolomide resistance $(28,29)$. However, to the best of our knowledge, the association between STAT3 and DIPG has not yet been elucidated.

In the present study, mRNA expression levels of various genes associated with the differentiation of NSCs (30) were compared between DIPG tissues and normal brain tissues using open public data. All of the screened genes, including Notch receptor 1 (NOTCH1), ACVR1 and STAT3, were significantly upregulated in DIPG tissues compared with in normal brain tissues. Based on its function in gliogenesis during neurodevelopment and in the oncogenesis of malignant tumors, including gliomas, the possible role of STAT3 in the tumor biology of DIPG was investigated using the human DIPG SF8628 cell line. STAT3 activation was modulated by treatment with the STAT3 inhibitor AG490 and transfection using STAT3 short hairpin (sh)RNA. Cell viability assays were performed to assess cell viability after modifying STAT3 activation. Protein expression was analyzed via western blotting and RNA expression was evaluated via reverse transcription-semi-quantitative PCR (RT-semi-qPCR). To investigate the effect of STAT3 inhibition on the therapeutic effect of irradiation, SF8628 cells were treated with a combination of STAT3 inhibition and irradiation.

\section{Materials and methods}

In silico $R 2$ analysis. Comparison of mRNA expression levels of genes associated with NSC differentiation [namely NOTCH1, inhibitor of DNA binding 1, ACVR1, Hes family bHLH transcription factor 1 (HES1), SMAD family member 1 , E1A binding protein p300, LIF receptor subunit $\alpha$ and STAT3] between normal brain tissues [ $\mathrm{n}=172$; Gene Expression Omnibus (GEO) ID: GSE11882 (31)] and DIPG tumor tissues [n=27, GEO ID: GSE26576 (32)] was performed using R2 and the Megasampler module (33): Genomics Analysis and Visualization Platform (http://r2.amc.nl), a microarray analysis and visualization platform. One-way ANOVA was used to compare the expression levels of STAT3 mRNA between the normal brain tissue and DIPG GEO datasets.
Cell culture. The patient-derived DIPG SF8628 cell line (Merck KGaA) harboring the histone H3.3 Lys 27-to-methionine (K27M) mutation was used in the present study. The cells were cultured in DMEM with high glucose supplemented with $10 \%$ FBS (both from Biowest) and 1\% penicillin/streptomycin (Gibco; Thermo Fisher Scientific, Inc.) at $37^{\circ} \mathrm{C}$ with $95 \%$ humidity and $5 \% \mathrm{CO}_{2}$.

STAT3 inhibitor treatment. To inhibit endogenous STAT3 activity, SF8628 cells were treated with $20 \mu \mathrm{M}$ of the STAT3 inhibitor AG490 (Cell Signaling Technology, Inc.) dissolved in DMSO.

Lentivirus-mediated shRNA silencing of STAT3 expression. Lentiviral shRNA particles were purchased from Sigma-Aldrich (Merck KGaA). Viral shRNA transfections using $10 \mu \mathrm{l}\left(1 \times 10^{7}\right.$ titer units $\left./ 0.1 \mathrm{ml}\right)$ STAT3-targeting shRNA or non-targeting shRNA were performed by incubating SF8628 cells in culture medium containing lentiviral particles for $12 \mathrm{~h}$ in the presence of $0.8 \mu \mathrm{g} / \mathrm{ml}$ polybrene (EMD Millipore). Subsequently, Puromycin (Thermo Fisher Scientific, Inc.) was added to the medium of the transfected cells to select shRNA-expressing cells.

$R T$-semi-qPCR. RT-semi-qPCR was performed to determine the transcript level of STAT3 in SF8628 cells, and the amplification of $\beta$-actin transcripts was used as the control to normalize the transcript levels of molecules. Total RNA was isolated from cells using TRIzol reagent (Invitrogen; Thermo Fisher Scientific, Inc.), and RT was performed to synthesize cDNA in a $20 \mu 1$ reaction mixture (SuperScript One-Step kit; Invitrogen; Thermo Fisher Scientific, Inc.) containing $2.5 \mu \mathrm{l}$ of each 10 pmol gene-specific primer, $1 \mu \mathrm{g}$ RNA, $2 \mathrm{X}$ reaction buffer, $0.4 \mu 1$ Taq polymerase, $0.4 \mathrm{mM}$ of each dNTP and $1.2 \mathrm{mM} \mathrm{MgCl}_{2}$, for $30 \mathrm{~min}$ at $50^{\circ} \mathrm{C}$. After an initial denaturation step of cDNA for 2 min at $94^{\circ} \mathrm{C}$, the cDNA of STAT3 transcripts was amplified for 25 cycles of $30 \mathrm{sec}$ at $94^{\circ} \mathrm{C}, 30 \mathrm{sec}$ at $58^{\circ} \mathrm{C}$ and $30 \mathrm{sec}$ at $72^{\circ} \mathrm{C}$, while the cDNA of $\beta$-actin transcripts was amplified for 18 cycles of $30 \mathrm{sec}$ at $94^{\circ} \mathrm{C}, 30 \mathrm{sec}$ at $52^{\circ} \mathrm{C}$ and $30 \mathrm{sec}$ at $70^{\circ} \mathrm{C}$. The PCR cycling numbers had been optimized to avoid amplification saturation. A total of $5 \mu \mathrm{l}$ qPCR product was separated on $1 \%$ agarose gels, which were subsequently stained with RedSafe ${ }^{\mathrm{TM}}$ Nucleic Acid Staining solution (Intron Biotechnology, Inc.). Primer sequences were as follows: STAT3 forward, 5'-ACCCAACAGCCGCCGTAG-3' and reverse, 5'-CAGATGGTTGTTTCCATTCAGAT-3'; and $\beta$-actin forward, 5'-ACACCTTCTACAATGAGCTG-3' and reverse, 5'-CATGATGGAGTTGAAGGTAG-3'.

Western blotting. Lysates of subconfluent (60-80\% confluent) cells were obtained using SDS lysis buffer [125 mM Tris-HCl (pH 6.8), $4 \%$ SDS, $0.004 \%$ bromophenol blue and $20 \%$ glycerol]. The Pierce BCA Protein assay kit (Thermo Fisher Scientific, Inc.) was used to determine protein concentration. The cell lysates (30 $\mu \mathrm{g}$ protein/lane) were separated by $10 \%$ SDS-PAGE and transferred to polyvinylidene difluoride membranes (EMD Millipore) that were blocked with 5\% skim dry milk in Tween-20 (0.05\%)-TBS (TTBS) for $1 \mathrm{~h}$ at room temperature. Subsequently, the membranes were incubated overnight at $4^{\circ} \mathrm{C}$ with primary antibodies $(1: 1,000)$ against 
one of the following: Cleaved caspase 3 (cat. no. ab2302; Abcam), cleaved poly (ADP-ribose) polymerase (cat. no. 9542; Cell Signaling Technology, Inc.), phosphorylated-STAT3 (pSTAT3; cat. no. 9131; Cell Signaling Technology, Inc.), STAT3 (cat. no. 9139; Cell Signaling Technology, Inc.), cyclin D1 (cat. no. MA5-16356; Thermo Fisher Scientific, Inc.), E-cadherin (cat. no. 610181; BD Biosciences), N-cadherin (cat. no. C3865; Sigma-Aldrich; Merck KGaA;), vimentin (cat. no. 3390S; Cell Signaling Technology, Inc.), Twist (cat. no. SC-15393; Santa Cruz Biotechnology, Inc.), Snail (cat. no. SC-28199; Santa Cruz Biotechnology, Inc.) and matrix metallopeptidase 9 (MMP-9; cat. no. RB-1539-P; NeoMarkers, Inc.). Horseradish peroxidase-conjugated anti-rabbit IgG (cat. no. bs-0295G-HRP) or anti-mouse IgG (cat. no. bs-0296G-HRP) (both 1:4,000; BIOSS) were used as secondary antibodies, and the membranes were incubated for $2 \mathrm{~h}$ at room temperature. Enhanced chemiluminescence (Invitrogen; Thermo Fisher Scientific, Inc.) was used to detect the immunoreactive proteins. $\beta$-actin (primary antibody, 1:1,000; cat. no. SC-47778; Santa Cruz Biotechnology, Inc.) served as the loading control.

Cell viability assay. Cell viability was assessed using a Cell Counting Kit (CCK)-8 assay (Dojindo Molecular Technologies, Inc.) according to the manufacturer's protocol. Briefly, $5 \times 10^{3}$ cells were seeded in each well of a 96-well plate and incubated for $48 \mathrm{~h}$ at $37^{\circ} \mathrm{C}$. The CCK- 8 reagent was added to each well $1 \mathrm{~h}$ before the incubation endpoint. Optical density values at $420 \mathrm{~nm}$ were determined using an ELISA plate reader (Bio-Rad Laboratories, Inc.).

Transwell migration and invasion assays. A 24-well Transwell Insert system with an $8-\mu \mathrm{m}$ pore size polyethylene terephthalate membrane was purchased from BD Biosciences. The upper chambers were coated with or without Matrigel for $1 \mathrm{~h}$ at $37^{\circ} \mathrm{C}$ for invasion or migration, respectively. Medium containing $10 \%$ FBS was placed in the lower chambers and served as a chemoattractant. SF8628 cells $\left(1 \times 10^{4}\right.$ cells/insert $)$ in $300 \mu 11 \%$ FBS-containing medium were seeded in the upper chamber of each Transwell insert and allowed to migrate for $48 \mathrm{~h}$ at $37^{\circ} \mathrm{C}$. Non-migrated cells were removed from the top of each insert with a cotton swab. Migrated cells on the bottom surface of the insert were stained with $0.2 \%$ crystal violet in $20 \%$ methanol for $30 \mathrm{~min}$ at room temperature and visualized with an inverted light microscope (magnification, $\mathrm{x} 40$ ). The stained cells were lysed in 10\% SDS for $30 \mathrm{~min}$, and the absorbance was measured at $562 \mathrm{~nm}$ using an ELISA plate reader.

F-actin assay. To examine whether STAT3 inhibition causes cytoskeletal reorganization, filamentous actin (F-actin) was visualized. Cells were incubated with $165 \mathrm{nmol} / \mathrm{l}$ Alexa Fluor-633-conjugated phalloidin (cat. no. A22284; Invitrogen; Thermo Fisher Scientific, Inc.) for $10 \mathrm{~min}$ at room temperature, followed by 4'6'-diamidio-2-phenoylindole (DAPI) staining for $10 \mathrm{~min}$ at room temperature. Immunofluorescence was observed by fluorescence microscopy (magnification, $\mathrm{x} 400$ ).

Combination of STAT3 inhibition and irradiation. Control cells and STAT3-inhibited cells (by shRNA transfection or
AG490 treatment) were cultured in 96-well plates at a density of $5 \times 10^{3} /$ well for $24 \mathrm{~h}$ at $37^{\circ} \mathrm{C}$ with $95 \%$ humidity and $5 \% \mathrm{CO}_{2}$. The cells were irradiated with $4 \mathrm{~Gy}$ using a 6-MV photon beam in a linear accelerator (21EX-S; Varian Medical Systems) and incubated for $24 \mathrm{~h}$ at $37^{\circ} \mathrm{C}$. Subsequently, a CCK- 8 assay was used as aforementioned to measure cell viability. All treatments were tested in triplicate.

Phosphorylated $H 2 A X$ variant histone $(\gamma H 2 A X)$ assay. Cells were cultured on a 4 -well chamber slide $\left(3 \times 10^{4}\right.$ cells/chamber). Following irradiation as aforementioned, cells were incubated for 1,4 or $24 \mathrm{~h}$, fixed in $4 \%$ paraformaldehyde for $10 \mathrm{~min}$ and permeabilized using $0.5 \%$ Triton $\mathrm{X}-100$ for $5 \mathrm{~min}$, both at room temperature. After blocking with 5\% skim milk diluted in $0.05 \%$ Triton X-100 for $30 \mathrm{~min}$ at room temperature, the slides were washed for $5 \mathrm{~min}$ in TTBS and incubated with a primary rabbit anti- $\gamma \mathrm{H} 2 \mathrm{AX}$ antibody (clone 2577; cat. no. 2577; Cell Signaling Technology, Inc.) at a 1:200 dilution in TTBS for $2 \mathrm{~h}$ at room temperature in a humidified chamber. Subsequently, the slides were washed three times in TTBS for $5 \mathrm{~min}$ each and incubated with an Alexa Fluor 488 goat anti-rabbit secondary IgG antibody (cat. no. A11008; Invitrogen; Thermo Fisher Scientific, Inc.) diluted 1:500 in 1\% BSA for $1 \mathrm{~h}$ at room temperature in a humidified chamber. The slides were washed twice in TTBS and mounted with DAPI-containing mounting medium. Images of $\gamma \mathrm{H} 2 \mathrm{AX}$ were acquired with a fluorescence microscope (magnification, x400; BX51; Olympus Corporation).

Statistical analysis. Associations between STAT3 activation and either the cell viability index, invasion or migration were analyzed using a two-tailed unpaired Student's t-test (for differences between two groups) using Excel 2016 (Microsoft Corporation) or one-way ANOVA (for multiple comparisons) followed by Tukey's post hoc test using SPSS v23 (IBM Corp.). Data are presented as the mean $\pm \mathrm{SD}(\mathrm{n} \geq 3)$. $\mathrm{P}<0.05$ was considered to indicate a statistically significant difference.

\section{Results}

In silico analysis identifies STAT3 as a potential oncogene in $D I P G$. The expression levels of astrogliogenesis-associated genes (30) between normal brain and DIPG tissues were compared using the publicly available microarray analysis platform R2 and the Megasampler module, previously described by Kumar et al (33). According to the expression analysis, all of these molecules were significantly upregulated in DIPG compared with in normal brain tissues (Fig. 1). Among the analyzed molecules, HES1 and STAT3 are transcription factors that regulate hallmarks of cancer $(34,35)$. Based on the results of a previous study (36) on the radiosensitizing effect of STAT3 inhibition in glioma, STAT3 was further investigated as a potential target to inhibit the oncogenic phenotype of DIPG cells.

STAT3 activation is associated with DIPG cell viability. To determine the oncogenic role of STAT3, the effect of STAT3 inactivation on the viability of SF8628 cells was examined via treatment with the STAT3 inhibitor AG490 or via STAT3 shRNA transfection. The transfections with shRNAs were 


\begin{tabular}{lllll}
\hline Aene & Probeset & $\begin{array}{l}\text { Expresssion } \\
\text { normal } \\
\text { brain }\left(\log _{2}\right)\end{array}$ & $\begin{array}{l}\text { Expresssion } \\
\text { DIPG }\left(\log _{2}\right)\end{array}$ & P-value \\
\hline NOTCH1 & 218902_at & 6.61 & 8.48 & $9.6 \times 10^{-40}$ \\
ID1 & 208937_s_at & 7.82 & 8.58 & $1.7 \times 10^{-5}$ \\
ACVR1 & 203935_at & 7.14 & 8.20 & $4.8 \times 10^{-18}$ \\
HES1 & 203934_s_at & 6.94 & 8.10 & $5.9 \times 10^{-9}$ \\
SMAD1 & 210993_s_at & 7.63 & 8.37 & $4.3 \times 10^{-21}$ \\
EP300 & 202221_s_at & 6.52 & 7.30 & $2.5 \times 10^{-10}$ \\
LIFR & 225575_at & 9.23 & 9.97 & $3.6 \times 10^{-9}$ \\
STAT3 & 208991_at & 9.37 & 10.04 & $1.1 \times 10^{-11}$ \\
\hline
\end{tabular}

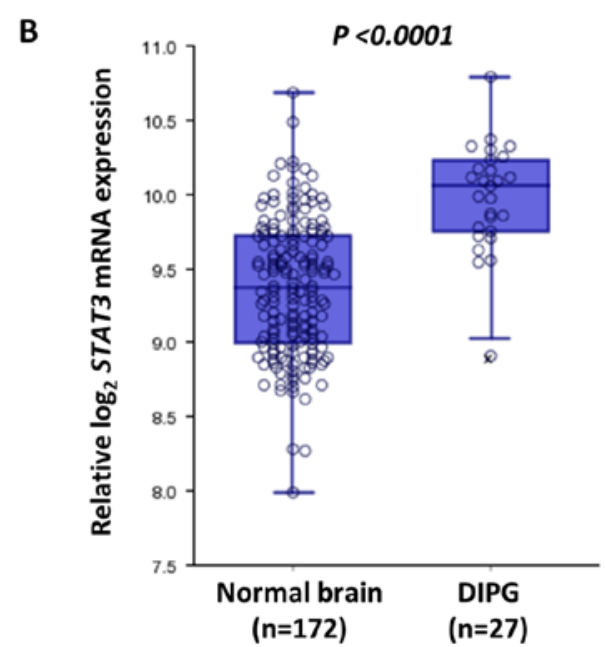

Figure 1. mRNA expression levels of astrogliogenesis-associated genes are high in DIPG. (A) In silico analysis of astrogliogenesis-associated gene mRNA expression in normal brain and DIPG tissues. (B) Relative STAT3 mRNA expression in normal brain and DIPG tissues. Each circle represents a tissue sample. DIPG, diffuse intrinsic pontine glioma; NOTCH1, Notch receptor 1; ID1, inhibitor of DNA binding 1; ACVR1, activin A receptor type I; HES1, Hes family bHLH transcription factor 1; SMAD1, SMAD family member 1; EP300, E1A binding protein p300; LIFR, LIF receptor subunit $\alpha$; STAT3, signal transducer and activator of transcription 3 .

confirmed by RT-semi-qPCR and gel electrophoresis (Fig. 2A). SF8628 DIPG cells were treated with various concentrations of AG490. Western blotting revealed that treatment of SF8628 cells with various concentrations of AG490 resulted in a substantial decrease in the protein expression of the active form of STAT3 (pSTAT3) in a dose-dependent manner, whereas the protein expression of total STAT3 was not changed (data not shown). In SF8628 cells treated with $30 \mu \mathrm{M}$ AG490, cell viability was significantly reduced compared with cells treated vehicle control (DMSO), and was similar to the viability of cells treated with $20 \mu \mathrm{M}$ AG490 (Fig. 2B). Therefore, $20 \mu \mathrm{M}$ AG490 was used in the following experiments. The CCK-8 assay revealed that the viability of AG490-treated SF8628 cells after $48 \mathrm{~h}$ was decreased compared with that of control vehicle-treated cells (Fig. 2C). Similar results were observed for cells expressing STAT3 shRNA (Fig. 2D). Since AG490 treatment did not change the status of cell apoptosis manifested by cleaved caspase 3 and cleaved poly (ADP-ribose) polymerase (data not shown) in SF8628 cells, it was hypothesized that decreased cell viability by STAT3 inactivation was not a result from increased cell apoptosis. To further examine the role of STAT3 in the viability of DIPG cells, the effect of STAT3 inhibition on the expression of a representative viability marker, cyclin D1, was analyzed. Western blotting revealed that cyclin D1 expression decreased after STAT3 inhibition using AG490 or STAT3 shRNA (Fig. 2E).

STAT3 regulates EMT, as well as the migration and invasion of DIPG cells. As the main oncogenic mechanism of STAT3 is the promotion of EMT (37), the effect of STAT3 inactivation in SF8628 cells on EMT, motility and invasiveness was investigated. Western blotting revealed that STAT3 inactivation increased E-cadherin (epithelial cell marker) expression, but decreased the expression levels of $\mathrm{N}$-cadherin, vimentin, Twist, Snail and MMP-9 (mesenchymal cell markers) (Fig. 3A). Therefore, the present data suggested a regulatory role of STAT3 in the mesenchymal transition in DIPG cells. To confirm that STAT3 may promote the mesenchymal transition in DIPG cells, actin organization was examined. Control SF8628 cells produced numerous filopodia-like extensions 
A

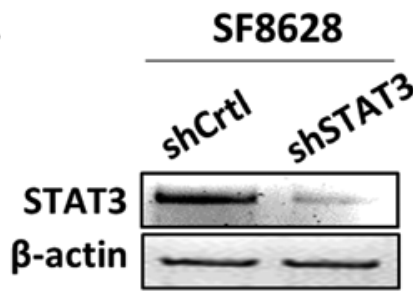

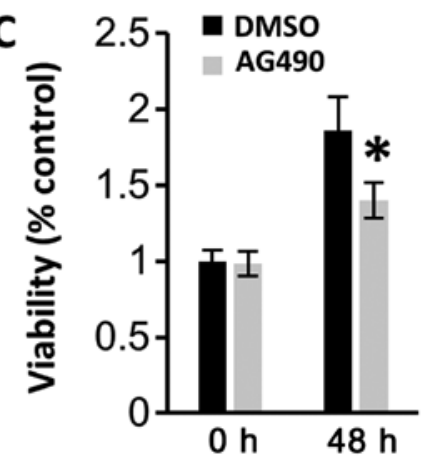

D
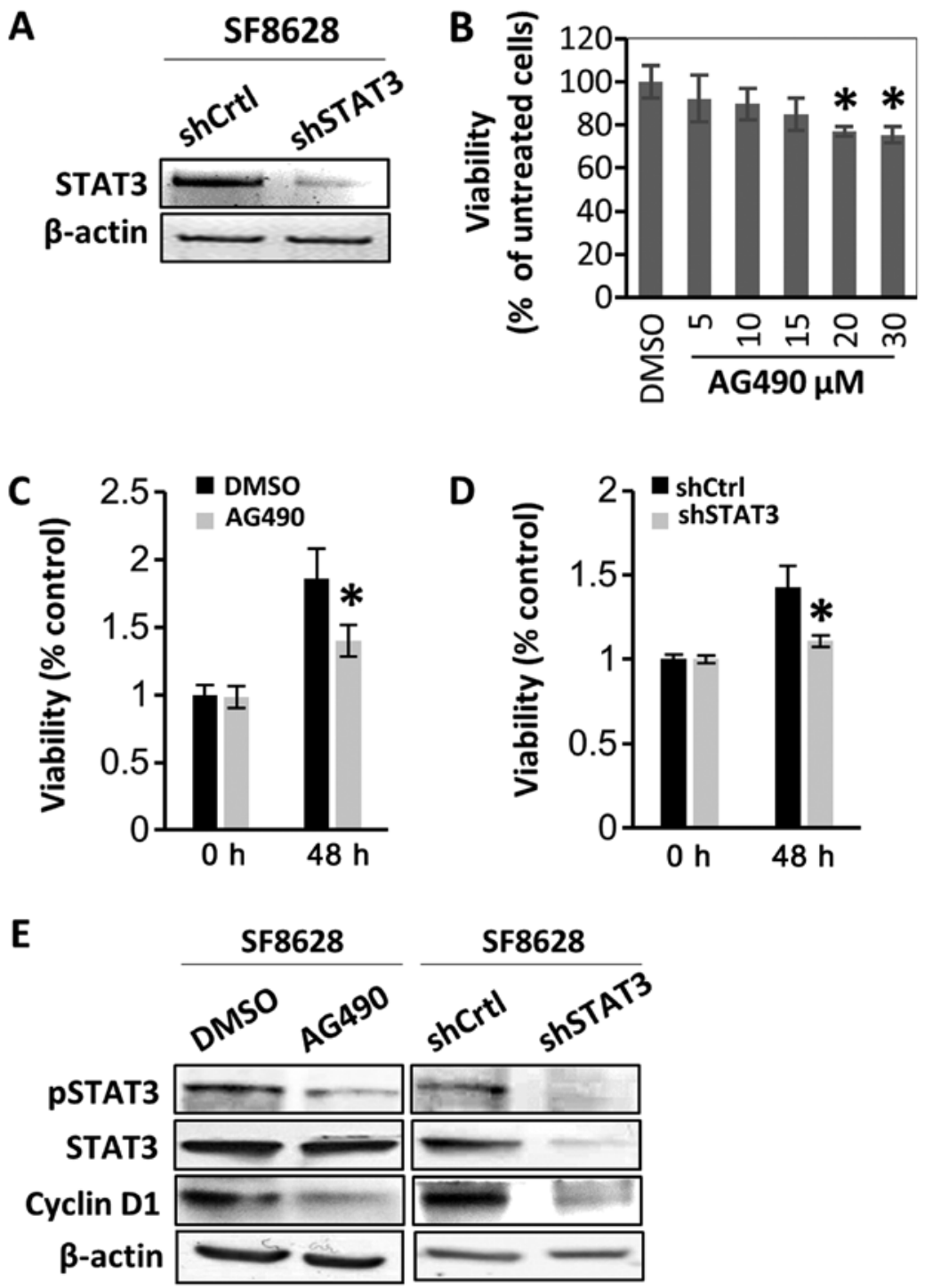

Figure 2. STAT3 inhibition suppresses human diffuse intrinsic pontine glioma cell viability. SF8628 cells were treated with vehicle control (DMSO) or AG490. SF8628 cells were transfected shCtrl or shSTAT3. (A) mRNA STAT3 expression was determined via reverse transcription-semi-quantitative PCR. $\beta$-actin mRNA was used as the loading control. (B) Cell viability data of AG490-treated cells. Cells were treated with various concentrations of AG490. Cell viability was analyzed using a CCK-8 assay, and absorbance was measured at $420 \mathrm{~nm}$. ${ }^{*} \mathrm{P}<0.05$ vs. DMSO-treated cells using one-way ANOVA. Data are presented as the mean \pm SD. (C and D) CCK-8 assay of control cells and STAT3-inhibited cells. Cell viability was analyzed using a CCK-8 assay, and absorbance was measured at $420 \mathrm{~nm} .{ }^{*} \mathrm{P}<0.05$ vs. DMSO-treated cells or shCtrl-transfected cells at $48 \mathrm{~h}$ using a two-tailed unpaired Student's t-test. Data are presented as the mean \pm SD. (E) Western blot analysis of pSTAT3, total STAT3 and cyclin D1. Protein samples were extracted from SF8628 control cells and STAT3-inhibited cells; $\beta$-actin was used as the loading control. Exposure time was 3 min for pSTAT3, 1 min for STAT3, 30 sec for cyclin D1 and 15 sec for $\beta$-actin. STAT3, signal transducer and activator of transcription 3; pSTAT3, phosphorylated STAT3; shCtrl, control short hairpin RNA; shSTAT3, STAT3 short hairpin RNA; CCK-8, Cell Counting Kit-8.

containing actin-rich bundles, which were absent or less visible in STAT3-inhibited cells (Fig. 3B). This suggested that the control cells exhibited more mesenchymal characteristics than the STAT3-inactivated cells. Migration and invasion assays were performed to assess the direct effect of STAT3 inhibition on cell motility and invasiveness. STAT3 inhibition significantly decreased cell migration and invasion (Fig. 3C and D, respectively), which further confirmed the potential effect of STAT3 on EMT induction in DIPG cells.

STAT3 inactivation increases the therapeutic effect of radiation. As STAT3 inhibitors have been reported to enhance radiation efficacy in various types of cancer (38-40), the present study investigated whether STAT3 inactivation was able to enhance the sensitivity of DIPG cells to radiation. SF8628 cells were exposed to $4 \mathrm{~Gy}$ of radiation $8 \mathrm{~h}$ after treatment with AG490 or DMSO. CCK- 8 assays $24 \mathrm{~h}$ after radiation indicated that viability was significantly decreased in cells treated with AG490 compared with that in cells treated with DMSO (Fig. 4A). Similar results were obtained when SF8628 cells expressing control shRNA or STAT3 shRNA were exposed to irradiation, which further suggests that STAT3 inhibition increases the therapeutic effect of radiation in DIPG cells (Fig. 4B).

Subsequently, the effect of STAT3 inhibition on the repair of radiation-induced DNA damage was examined using the DNA double strand break marker $\gamma \mathrm{H} 2 \mathrm{AX}$ at 1,4 and $24 \mathrm{~h}$ after treatment with 4 Gy radiation. As shown in Fig. 4C, $\gamma \mathrm{H} 2 \mathrm{AX}$ was localized in the nucleus in both AG490-treated 
A

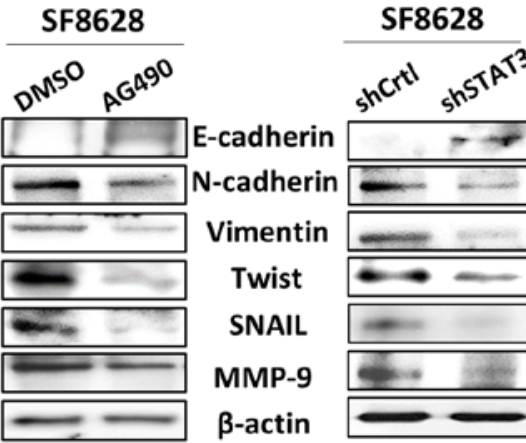

B

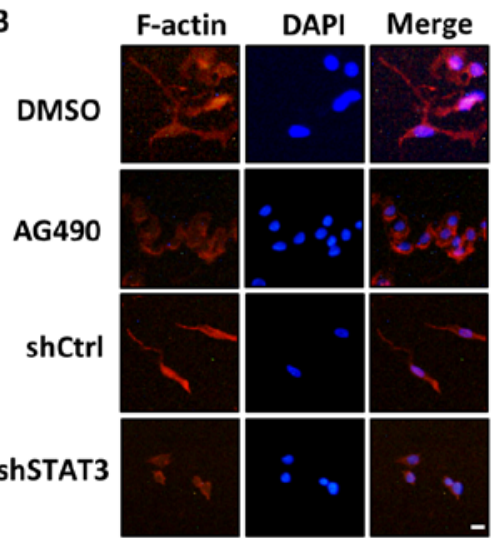

C
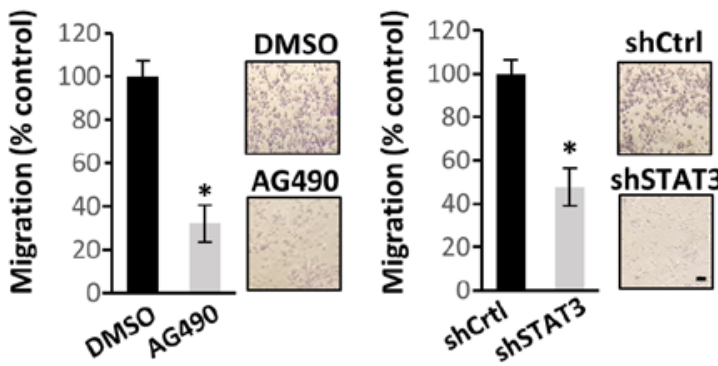

D
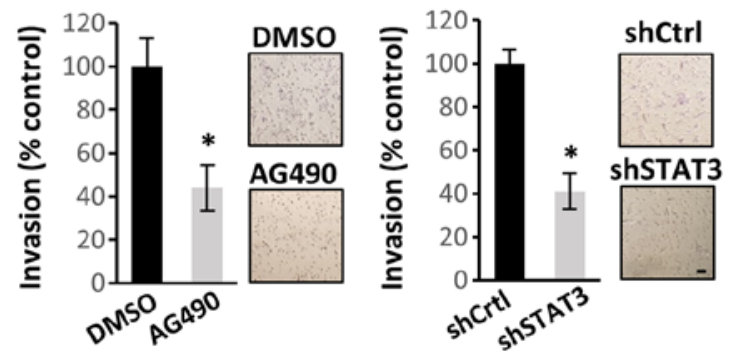

Figure 3. STAT3 inhibition suppresses human diffuse intrinsic pontine glioma cell migration and invasion by regulating EMT. SF8628 cells were treated with vehicle control (DMSO) or AG490. SF8628 cells were transfected with shCtrl or shSTAT3. (A) Western blot analysis of EMT markers. Protein samples were extracted from SF8628 control cells and STAT3-inhibited cells. Protein samples were tested for E-cadherin, N-cadherin, Vimentin, Twist, Snail, MMP-9 and $\beta$-actin (loading control) expression. Exposure time was $5 \mathrm{~min}$ for E-cadherin, 1 min for N-cadherin, Twist and Snail, 10 sec for Vimentin, 3 min for MMP-9 and $15 \mathrm{sec}$ for $\beta$-actin. (B) Organization of the actin cytoskeleton was determined by immunofluorescence staining. Alexa Fluor 633-conjugated phalloidin was used to visualize F-actin (red), and DAPI staining (blue) was used for visualization of cell nuclei. Original magnification, $\mathrm{x} 400$. Scale bar, $2 \mu \mathrm{m}$. (C) Cells were seeded in the upper chamber of Transwell inserts, and the cell migration ability was assessed $48 \mathrm{~h}$ after cell plating. Magnification, $\mathrm{x} 40$. Scale bar, $100 \mu \mathrm{m}$. (D) Cells were seeded in the upper chamber of Transwell inserts coated with Matrigel, and the cell invasion ability was measured $48 \mathrm{~h}$ after cell plating. Magnification, $\mathrm{x} 40$. Scale bar, $100 \mu \mathrm{m}$. The results were calculated as percentages relative to control cells. Representative images of invasive cells are shown next to each bar graph. Data are presented as the mean $\pm \mathrm{SD}$. "P $<0.05$ using a two-tailed unpaired Student's t-test. STAT3, signal transducer and activator of transcription 3; shCtrl, control short hairpin RNA; shSTAT3, STAT3 short hairpin RNA; EMT, epithelial-mesenchymal transition; MMP-9, matrix metallopeptidase-9; DAPI, 4'6'-diamidio-2-phenoylindole.

and control cells after $1 \mathrm{~h}$; these results indicated that DNA damage occurred early in both AG490-treated and control cells after radiation treatment. However, decreased expression of $\gamma \mathrm{H} 2 \mathrm{AX}$ was observed over time in cells treated with DMSO and radiation, but not in those treated with AG490 and radiation, indicating that repair of DNA damage predominantly occurred in control cells treated with radiation (Fig. 4C). Similar results were observed in cells transfected with STAT3 shRNA. Control shRNA-expressing cells treated with radiation exhibited lower $\gamma \mathrm{H} 2 \mathrm{AX}$ positivity than STAT3 shRNA-expressing cells at $24 \mathrm{~h}$ after radiation treatment (Fig. 4D). The present results suggest that STAT3 inhibition enhances the radiation sensitivity of DIPG cells.

\section{Discussion}

Recent technical advances have allowed the investigation of the concept that DIPG occurrence is associated with neural differentiation (41). As STAT3 is often upregulated in numerous types of cancer and serves a role in astrocyte differentiation, the present study investigated the effect of STAT3 inhibition on the oncogenic behavior of the DIPG SF8628 cell line. First, it was observed that STAT3 expression was upregulated in
DIPG tissues compared with normal brain tissues. Second, the effect of STAT3 upregulation was assessed by inhibition of STAT3 using shRNA or the STAT3 inhibitor AG490, and it was revealed that cell viability, migration and invasion were decreased as STAT3 was inhibited. Additionally, STAT3 inhibition induced changes opposite to those observed in EMT, such as an upregulated E-cadherin expression and downregulated Snail and MMP9 expression. Furthermore, the degree of cellular actin remodeling was decreased, suggesting the possibility that STAT3 may contribute to EMT in DIPG cells. Finally, the therapeutic potential of STAT3 inhibition was assessed in combination with radiation, which is the current mode of treatment. Inhibition of STAT3 in DIPG cells decreased their viability, as well as their DNA repair capacity. Although the current study contains the limitation of using a single DIPG cell line, the present results are consistent with previous reports on the role of STAT3 in cell viability, migration, prognosis and therapeutic resistance in glioma $(28,29)$.

STAT3 is one of the major potential targets that may be applied in molecular targeted therapy due to its known regulatory effects on oncogenic proteins. For example, STAT3 transcriptionally regulates cyclin D1 (42) and mesenchymal cell markers (37), such as Slug, Snail and Twist, which drive 

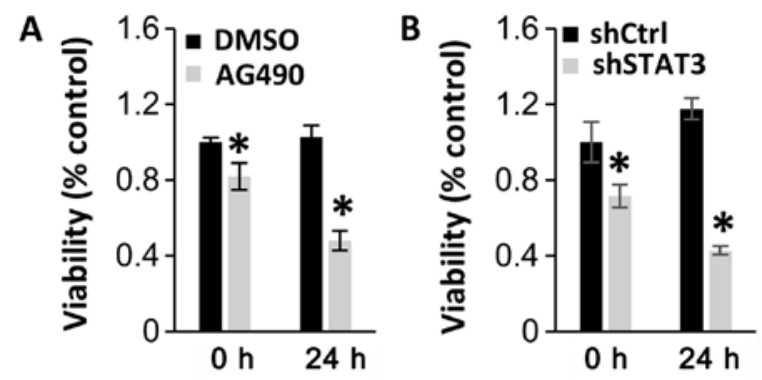

C

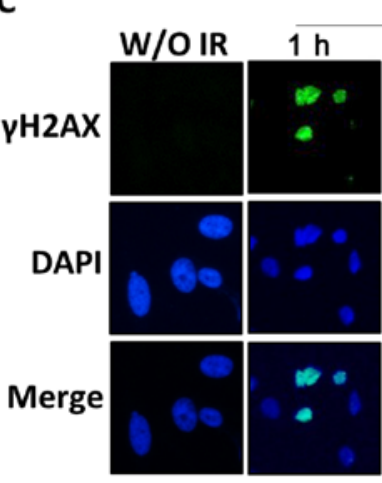

DMSO
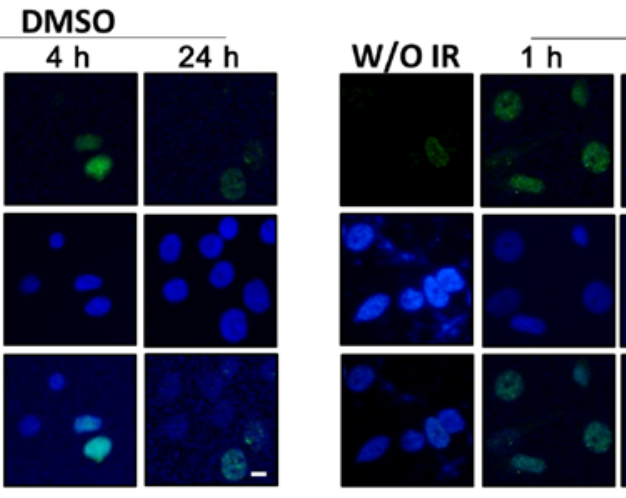

AG490
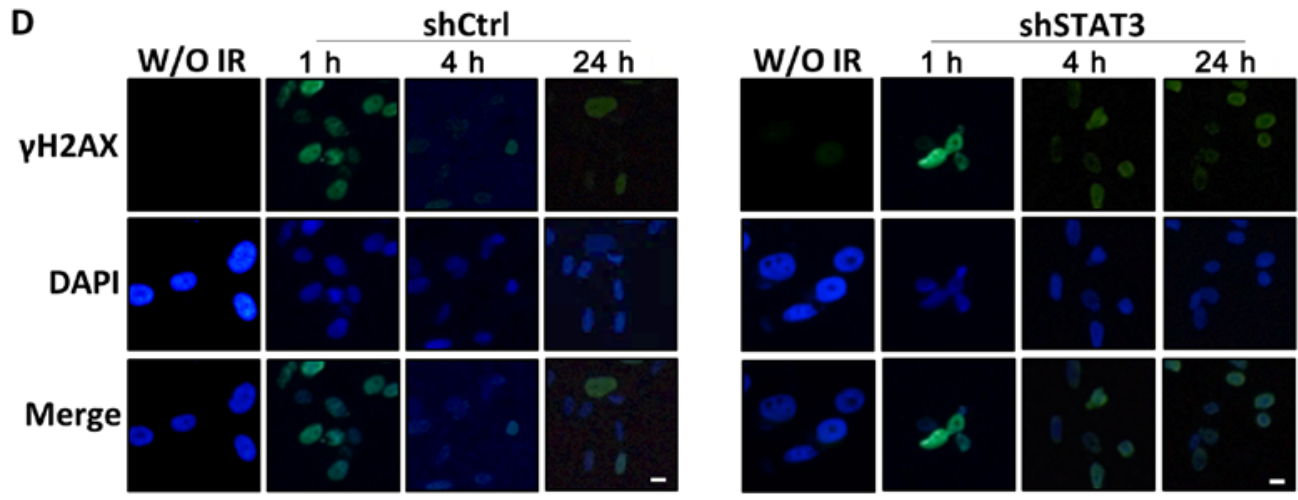

Figure 4. STAT3 inhibition sensitizes human diffuse intrinsic pontine glioma cells to radiation by interfering with DNA damage repair. SF8628 cells were treated with control vehicle (DMSO) or AG490. SF8628 cells were transfected with shCtrl or shSTAT3. (A and B) CCK-8 assays of control cells and STAT3-inhibited cells at 0 and $24 \mathrm{~h}$ after radiation treatment. Cell viability was analyzed using a CCK- 8 assay, and absorbance was measured at $420 \mathrm{~nm}$. ${ }^{*}<0.05$ using one-way ANOVA. Data are presented as the mean \pm SD. (C and D) Analysis of DNA damage repair after treatment with 4 Gy radiation by visualizing the double-strand marker $\gamma \mathrm{H} 2 \mathrm{AX}$. The panel shows representative images of $\gamma \mathrm{H} 2 \mathrm{AX}$ (green), DAPI-stained nuclei (blue) and merged images of SF8628 cells without irradiation and at 1, 4 and $24 \mathrm{~h}$ after radiation. Scale bar, $1 \mu \mathrm{m}$. STAT3, signal transducer and activator of transcription 3; shCtrl, control short hairpin RNA; shSTAT3, STAT3 short hairpin RNA; CCK-8, Cell Counting Kit-8; $\gamma \mathrm{H} 2 \mathrm{AX}$, phosphorylated H2A X variant histone; DAPI, 4'6'-diamidio-2-phenoylindole.

transformation and ultimately metastasis. In the present study, STAT3 inactivation decreased cyclin D1 expression and SF8628 cell viability. However, the current data did not reveal the transcriptional mechanism underlying cyclin D1 regulation by STAT3; nonetheless, based on previous studies $(42,43)$, it is hypothesized that STAT3 may act as a transactivator of cyclin D1 transcription in DIPG cells.

EMT confers migration and invasion abilities, and induces molecular phenotype changes in cancer cells (44). In addition, various types of cancer that are resistant to cytotoxic therapy are prone to displaying a mesenchymal phenotype (45). For example, chemoresistance has been attributed to EMT in adult glioblastoma $(46,47)$. Since EMT is one of the primary changes induced by STAT3 in cancer, the present study evaluated the effect of STAT3 inhibition in DIPG cells in association with this process. STAT3 inhibition decreased the migration and invasiveness of DIPG cells. Furthermore, STAT3 inhibition upregulated the expression levels of E-cadherin, an epithelial cell marker, and downregulated the expression levels of mesenchymal cell markers, indicating a change toward an epithelial phenotype. Therefore, the present results suggest that STAT3 contributes to the mesenchymal phenotype of DIPG through EMT, consistent with evidence supporting the possible role of the JAK/STAT signaling pathway in the mesenchymal transition in pediatric glioma (48). Although the JAK/STAT3/TWIST signaling pathway is involved in neurogenesis and astrogliogenesis under normal conditions (49), aberrant activation of this pathway may induce EMT in cancer $(50,51)$.

The STAT3 signaling pathway is known to serve a role in radioresistance in numerous types of cancer, including squamous cell carcinoma and head and neck carcinoma (52), and similar results have been reported for high-grade gliomas $(53,54)$. A previous in vitro and in vivo study has 
demonstrated that a STAT3 inhibitor potentiates the radiosensitizing effect of temozolomide in glioblastoma (55). In particular, previous studies $(56,57)$ have reported that STAT3-induced EMT mediates radioresistance in esophageal squamous carcinoma and glioblastoma. In esophageal squamous carcinoma, IL6/STAT3/TWIST signaling pathway-mediated EMT confers radioresistance both in vitro and in vivo (56). Additionally, the STAT3/Slug axis induces the EMT phenotype and radioresistance (57). A possible mechanism underlying STAT3/EMT/radioresistance is hypothesized to be associated with the role of STAT3 in DNA damage repair. Several studies have indicated that radiation-induced ataxia telangiectasia mutated, which is a key molecule initiating DNA damage repair upon radiation (58), positively regulates STAT3 via an unknown mechanism $(59,60)$. Therefore, STAT3 may serve a role in DNA damage repair mechanisms and mediate radiation resistance. In the present study, the combination of STAT3 inhibition and radiation resulted in significant combinatorial efficacy in DIPG cells. Therefore, the present data support STAT3 inhibition as a potential treatment in clinical studies of DIPG, but further investigations are required to clarify the precise mechanism underlying radiation resistance.

The role of STAT3 in neural development may reflect its participation in the oncogenesis of DIPG. It has been demonstrated that activation of STAT signaling via gp130 in cooperation with bone morphogenetic protein (BMP) signaling leads to the formation of the STAT3/SMAD/p300 complex on the astrogliogenic gene promoter (61) and inhibits oligodendrocyte progenitor cell differentiation into mature oligodendrocytes (62). Notably, $25 \%$ of DIPGs display activating mutations in ACVR1, which encodes the activin receptor-like kinase-2 in the BMP signaling pathway. Furthermore, the present study revealed that expression levels of principal astrogliogenesis-inducing proteins were mostly increased in DIPG compared with in normal brain. Overall, it can be hypothesized that increased STAT3 expression, in combination with the overexpression of BMP signaling pathway components caused by ACVR1 mutations, may cause excessive astrogliogenesis. Accordingly, malfunction of the BMP signaling pathway, including its key molecules ACVR1 and STAT3, may be a key driver in the development of DIPG with an astrocytic phenotype.

In conclusion, the present study revealed that STAT3 was upregulated in human DIPG samples compared with in normal brain samples, and that STAT3 inhibition decreased the oncogenic phenotype of DIPG cells. Furthermore, STAT3 inhibition enhanced the therapeutic effects of radiation therapy in DIPG cells. Therefore, STAT3 may serve as a therapeutic target for DIPG.

\section{Acknowledgements}

Not applicable.

\section{Funding}

The present study was supported by the National Research Foundation of Korea Grant funded by the Korean Government (grant no. NRF-2018R1A5A2025964), the Bio \& Medical Technology Development Program of the National Research
Foundation of Korea funded by the Ministry of Science \& Information and Communications Technology (grant no. NRF-2018-M3A9H3021707) and the Seoul National University Hospital Research Fund (grant no. 03-2016-0350).

\section{Availability of data and materials}

The datasets used and/or analyzed during the current study are available from the corresponding author on reasonable request. The datasets GSE11882 (https://www.ncbi.nlm. nih.gov/nucleotide?cmd=search\&term=GSE11882) and GSE26576 (https://www.ncbi.nlm.nih.gov/geo/query/acc. cgi?acc=GSE26576) were analyzed using the Genomics Analysis and Visualization platform (http://r2.amc.nl).

\section{Authors' contributions}

JP and JYL designed the study and wrote the original draft. JP was in charge of all experiments. SY, SPK, WL, KHK, JIK, SKK and KCW interpreted the data and revised the manuscript. All authors read and approved the final manuscript.

\section{Ethics approval and consent to participate}

Not applicable.

\section{Patient consent for publication}

Not applicable.

\section{Competing interests}

The authors declare that they have no competing interests.

\section{References}

1. Warren KE: Diffuse intrinsic pontine glioma: Poised for progress. Front Oncol 2: 205, 2012.

2. Khatua S, Sadighi ZS, Pearlman ML, Bochare S and Vats TS: Brain tumors in children-current therapies and newer directions. Indian J Pediatr 79: 922-927, 2012

3. Vanan MI and Eisenstat DD: DIPG in Children-what can we learn from the past? Front Oncol 5: 237, 2015.

4. Jansen MH, Van Vuurden DG, Vandertop WP and Kaspers GJ: Diffuse intrinsic pontine gliomas: A systematic update on clinical trials and biology. Cancer Treat Rev 38: 27-35, 2012.

5. Pollack IF, Jakacki RI, Blaney SM, Hancock ML, Kieran MW, Phillips P, Kun LE, Friedman H, Packer R, Banerjee A, et al: Phase I trial of imatinib in children with newly diagnosed brainstem and recurrent malignant gliomas: A pediatric brain tumor consortium report. Neuro Oncol 9: 145-160, 2007.

6. Geoerger B, Hargrave D, Thomas F, Ndiaye A, Frappaz D, Andreiuolo F, Varlet P, Aerts I, Riccardi R, Jaspan T, et al: Innovative therapies for Children with cancer pediatric phase I study of erlotinib in brainstem glioma and relapsing/refractory brain tumors. Neuro Oncol 13: 109-118, 2011.

7. Wu G, Diaz AK, Paugh BS, Rankin SL, Ju B, Li Y, Zhu X, Qu C, Chen X, Zhang J, et al: The genomic landscape of diffuse intrinsic pontine glioma and pediatric non-brainstem high-grade glioma. Nat Genet 46: 444-450, 2014.

8. Hall A, Giese NA and Richardson WD: Spinal cord oligodendrocytes develop from ventrally derived progenitor cells that express PDGF alpha-receptors. Development 122: 4085-4094, 1996.

9. Verschueren K, Dewulf N, Goumans MJ, Lonnoy O, Feijen A, Grimsby S, Vandi Spiegle K, ten Dijke P, Morén A, Vanscheeuwijck P, et al: Expression of type I and type IB receptors for activin in midgestation mouse embryos suggests distinct functions in organogenesis. Mech Dev 52: 109-123, 1995. 
10. Buczkowicz P, Bartels U, Bouffet E, Becher O and Hawkins $C$ : Histopathological spectrum of paediatric diffuse intrinsic pontine glioma: Diagnostic and therapeutic implications. Acta Neuropathol 128: 573-581, 2014.

11. Saratsis AM, Kambhampati M, Snyder K, Yadavilli S, Devaney JM, Harmon B, Hall J, Raabe EH, An P, Weingart M, et al: Comparative multidimensional molecular analyses of pediatric diffuse intrinsic pontine glioma reveals distinct molecular subtypes. Acta Neuropathol 127: 881-895, 2014.

12. Achanta P, Sedora Roman NI and Quiñones-Hinojosa A: Gliomagenesis and the use of neural stem cells in brain tumor treatment. Anticancer Agents Med Chem 10: 121-130, 2010.

13. Levy DE and Lee CK: What does Stat3 do? J Clin Invest 109: 1143-1148, 2002

14. Duncan SA, Zhong Z, Wen Z and Darnell JE Jr: STAT signaling is active during early mammalian development. Dev Dyn 208 190-198, 1997.

15. Wegenka UM, Buschmann J, Lütticken C, Heinrich PC and Horn F: Acute-phase response factor, a nuclear factor binding to acute-phase response elements, is rapidly activated by interleukin-6 at the posttranslational level. Mol Cell Biol 13: 276-288, 1993.

16. Levy DE and Darnell JE Jr: Stats: Transcriptional control and biological impact. Nat Rev Mol Cell Biol 3: 651-662, 2002.

17. Fan G, Martinowich K, Chin MH, He F, Fouse SD, Hutnick L, Hattori D, Ge W, Shen Y, Wu H, et al: DNA methylation controls the timing of astrogliogenesis through regulation of JAK-STAT signaling. Development 132: 3345-3356, 2005.

18. Ma JH, Qin L and Li X: Role of STAT3 signaling pathway in breast cancer. Cell Commun Signal 18: 33, 2020.

19. Dutta P, Sabri N, Li J and Li WX: Role of STAT3 in lung cancer JAKSTAT 3: e999503, 2014.

20. Corcoran RB, Contino G, Deshpande V, Tzatsos A, Conrad C, Benes CH, Levy DE, Settleman J, Engelman JA and Bardeesy N: STAT3 plays a critical role in KRAS-induced pancreatic tumorigenesis. Cancer Res 71: 5020-5029, 2011.

21. Yu CL, Meyer DJ, Campbell GS, Larner AC, Carter-Su C, Schwartz J and Jove R: Enhanced DNA-binding activity of a Stat3-related protein in cells transformed by the Src oncoprotein. Science 269: 81-83, 1995

22. Garcia R, Yu CL, Hudnall A, Catlett R, Nelson KL, Smithgall T, Fujita DJ, Ethier SP and Jove R: Constitutive activation of Stat3 in fibroblasts transformed by diverse oncoproteins and in breast carcinoma cells. Cell Growth Differ 8: 1267-1276, 1997.

23. Lund TC, Prator PC, Medveczky MM and Medveczky PG: The Lck binding domain of herpesvirus saimiri tip-484 constitutively activates Lck and STAT3 in T cells. J Virol 73: 1689-1694 1999.

24. Wen X, Lin HH, Shih HM, Kung HJ and Ann DK: Kinase activation of the non-receptor tyrosine kinase Etk/BMX alone is sufficient to transactivate STAT-mediated gene expression in salivary and lung epithelial cells. J Biol Chem 274: 38204-38210, 1999.

25. West AJ, Tsui V, Stylli SS, Nguyen HPT, Morokoff AP, Kaye AH and Luwor RB: The role of interleukin-6-STAT3 signalling in glioblastoma. Oncol Lett 16: 4095-4104, 2018.

26. Ganguly D, Fan M, Yang CH, Zbytek B, Finkelstein D, Roussel MF and Pfeffer LM: The critical role that STAT3 plays in glioma-initiating cells: STAT3 addiction in glioma. Oncotarget 9: 22095-22112, 2018.

27. Kim JE, Patel M, Ruzevick J, Jackson CM and Lim M: STAT3 Activation in glioblastoma: Biochemical and therapeutic implications. Cancers (Basel) 6: 376-395, 2014.

28. Ouédraogo ZG, Biau J, Kemeny JL, Morel L, Verrelle P and Chautard E: Role of STAT3 in genesis and progression of human malignant gliomas. Mol Neurobiol 54: 5780-5797, 2017.

29. Chang N, Ahn SH, Kong DS, Lee HW and Nam DH: The role of STAT3 in glioblastoma progression through dual influences on tumor cells and the immune microenvironment. Mol Cell Endocrinol 451: 53-65, 2017.

30. Chuang JH, Tung LC and Lin Y: Neural differentiation from embryonic stem cells in vitro: An overview of the signaling pathways. World J Stem Cells 7: 437-447, 2015.

31. Berchtold NC, Cribbs DH, Coleman PD, Rogers J, Head E, Kim R, Beach T, Miller C, Troncoso J, Trojanowski JQ, et al: Gene expression changes in the course of normal brain aging are sexually dimorphic. Proc Natl Acad Sci USA 105: 15605-15610, 2008.
32. Paugh BS, Broniscer A, Qu C, Miller CP, Zhang J, Tatevossian RG, Olson JM, Geyer JR, Chi SN, da Silva NS, et al: Genome-wide analyses identify recurrent amplifications of receptor tyrosine kinases and cell-cycle regulatory genes in diffuse intrinsic pontine glioma. J Clin Oncol 29: 3999-4006, 2011.

33. Kumar SS, Sengupta S, Lee K, Hura N, Fuller C, DeWire M, Stevenson CB, Fouladi M and Drissi R: BMI-1 is a potential therapeutic target in diffuse intrinsic pontine glioma. Oncotarget 8 : 62962-62975, 2017.

34. Liu ZH, Dai XM and Du B: Hes1: A key role in stemness, metastasis and multidrug resistance. Cancer Biol Ther 16: 353-359, 2015.

35. Kamran MZ, Patil P and Gude RP: Role of STAT3 in cancer metastasis and translational advances. Biomed Res Int 2013: $421821,2013$.

36. Yang YP, Chang YL, Huang PI, Chiou GY, Tseng LM, Chiou SH, Chen MH, Chen MT, Shih YH, Chang CH, et al: Resveratrol suppresses tumorigenicity and enhances radiosensitivity in primary glioblastoma tumor initiating cells by inhibiting the STAT3 axis. J Cell Physiol 227: 976-993, 2012.

37. Wendt MK, Balanis N, Carlin CR and Schiemann WP STAT3 and epithelial-mesenchymal transitions in carcinomas. JAKSTAT 3: e28975, 2014.

38. Lau J,Ilkhanizadeh S, Wang S, Miroshnikova YA, Salvatierra NA, Wong RA, Schmidt C, Weaver VM, Weiss WA and Persson AI: STAT3 blockade inhibits radiation-induced malignant progression in glioma. Cancer Res 75: 4302-4311, 2015.

39. Bharadwaj U, Eckols TK, Xu X, Kasembeli MM, Chen Y, Adachi M, Song Y,Mo Q,Lai SY and Tweardy DJ: Small-molecule inhibition of STAT3 in radioresistant head and neck squamous cell carcinoma. Oncotarget 7: 26307-26330, 2016.

40. You S, Li R, Park D, Xie M, Sica GL, Cao Y, Xiao ZQ and Deng X: Disruption of STAT3 by niclosamide reverses radioresistance of human lung cancer. Mol Cancer Ther 13: 606-616, 2014.

41. Puget S, Philippe C, Bax DA, Job B, Varlet P, Junier MP, Andreiuolo F, Carvalho D, Reis R, Guerrini-Rousseau L, et al: Mesenchymal transition and PDGFRA amplification/mutation are key distinct oncogenic events in pediatric diffuse intrinsic pontine gliomas. PLoS One 7: e30313, 2012.

42. Leslie K, Lang C, Devgan G, Azare J, Berishaj M, Gerald W, Kim YB, Paz K, Darnell JE, Albanese C, et al: Cyclin D1 is transcriptionally regulated by and required for transformation by activated signal transducer and activator of transcription 3 . Cancer Res 66: 2544-2552, 2006.

43. Gu J, Li G, Sun T, Su Y, Zhang X, Shen J, Tian Z and Zhang J: Blockage of the STAT3 signaling pathway with a decoy oligonucleotide suppresses growth of human malignant glioma cells. J Neurooncol 89: 9-17, 2008.

44. Brabletz T, Kalluri R, Nieto MA and Weinberg RA: EMT in cancer. Nat Rev Cancer 18: 128-134, 2018.

45. Shintani Y, Okimura A, Sato K, Nakagiri T, Kadota Y, Inoue M, Sawabata N, Minami M, Ikeda N, Kawahara K, et al: Epithelial to mesenchymal transition is a determinant of sensitivity to chemoradiotherapy in non-small cell lung cancer. Ann Thorac Surg 92: 1794-1804, 2011.

46. Siebzehnrubl FA, Silver DJ, Tugertimur B, Deleyrolle LP, Siebzehnrubl D, Sarkisian MR, Devers KG, Yachnis AT, Kupper MD, Neal D, et al: The ZEB1 pathway links glioblastoma initiation, invasion and chemoresistance. EMBO Mol Med 5: 1196-1212, 2013

47. Kahlert UD, Nikkhah G and Maciaczyk J: Epithelial-tomesenchymal(-like) transition as a relevant molecular event in malignant gliomas. Cancer Lett 331: 131-138, 2013.

48. Meel MH, Schaper SA, Kaspers GJL and Hulleman E: Signaling pathways and mesenchymal transition in pediatric high-grade glioma. Cell Mol Life Sci 75: 871-887, 2018.

49. Cao F, Hata R, Zhu P, Nakashiro K and Sakanaka M: Conditional deletion of Stat 3 promotes neurogenesis and inhibits astrogliogenesis in neural stem cells. Biochem Biophys Res Commun 394: 843-847, 2010.

50. Uddin N, Kim RK, Yoo KC, Kim YH, Cui YH, Kim IG, Suh Y and Lee SJ: Persistent activation of STAT3 by PIM2-driven positive feedback loop for epithelial-mesenchymal transition in breast cancer. Cancer Sci 106: 718-725, 2015.

51. Xiong H, Hong J, Du W, Lin YW, Ren LL, Wang YC, Su WY, Wang JL, Cui Y, Wang ZH and Fang JY: Roles of STAT3 and ZEB1 proteins in E-cadherin down-regulation and human colorectal cancer epithelial-mesenchymal transition. J Biol Chem 287: 5819-5832, 2012. 
52. Han X, Xue X, Zhou H and Zhang G: A molecular view of the radioresistance of gliomas. Oncotarget 8: 100931-100941, 2017.

53. Gao L, Li F, Dong B, Zhang J, Rao Y, Cong Y, Mao B and Chen X: Inhibition of STAT3 and ErbB2 suppresses tumor growth, enhances radiosensitivity, and induces mitochondria-dependent apoptosis in glioma cells. Int J Radiat Oncol Biol Phys 77: $1223-1231,2010$.

54. Yuan X, Du J, Hua S, Zhang H, Gu C, Wang J, Yang L, Huang J, $\mathrm{Yu}$ J and Liu F: Suppression of autophagy augments the radiosensitizing effects of STAT3 inhibition on human glioma cells. Exp Cell Res 330: 267-276, 2015.

55. Han TJ, Cho BJ, Choi EJ, Kim DH, Song SH, Paek SH and Kim IA: Inhibition of STAT3 enhances the radiosensitizing effect of temozolomide in glioblastoma cells in vitro and in vivo. J Neurooncol 130: 89-98, 2016.

56. Zang C, Liu X, Li B, He Y, Jing S, He Y, Wu W, Zhang B, Ma S, Dai W, et al: IL-6/STAT3/TWIST inhibition reverses ionizing radiation-induced EMT and radioresistance in esophageal squamous carcinoma. Oncotarget 8: 11228-11238, 2017.
57. Lin JC, Tsai JT, Chao TY, Ma HI and Liu WH: The STAT3/Slug axis enhances radiation-induced tumor invasion and cancer stem-like properties in radioresistant glioblastoma. Cancers (Basel) 10: pii: E512, 2018.

58. Guleria A and Chandna S: ATM kinase: Much more than a DNA damage responsive protein. DNA Repair (Amst) 39: 1-20, 2016.

59. Shen M, Xu Z, Xu W, Jiang K, Zhang F, Ding Q, Xu Z and Chen Y: Inhibition of ATM reverses EMT and decreases metastatic potential of cisplatin-resistant lung cancer cells through JAK/STAT3/PD-L1 pathway. J Exp Clin Cancer Res 38: 149, 2019.

60. Zhang Y, Cho YY,Petersen BL, Bode AM,Zhu F and Dong Z: Ataxia telangiectasia mutated proteins, MAPKs, and RSK2 are involved in the phosphorylation of STAT3. J Biol Chem 278: 12650-12659, 2003.

61. Shimazaki T and Okano H: Heterochronic microRNAs in temporal specification of neural stem cells: Application toward rejuvenation. NPJ Aging Mech Dis 2: 15014, 2016.

62. Samanta J and Kessler JA: Interactions between ID and OLIG proteins mediate the inhibitory effects of BMP4 on oligodendroglial differentiation. Development 131: 4131-4142, 2004. 